\title{
A DYNAMIC PROGRAMMING GENERALIZATION OF $x y$ TO $n$ VARIABLES
}

A. P. HillmaN, D. G. MEAD, K. B. O'KeEFE ${ }^{1}$ AND E. S. O'KeEFE

A function $\phi\left(d_{1}, \cdots, d_{n}\right)$, from the ordered $n$-tuples of nonnegative integers into the nonnegative integers augmented by $\infty$, with the following properties arises in the investigation [6] of the differential ideal generated by a product of $n$ differential indeterminates:

(A) $\phi\left(d_{1}, \cdots, d_{n}\right)$ is symmetric in the $d_{i}$.

(B) $\phi(0)=0$ and $\phi(x)=\infty$ for $x>0$.

(C) $\phi(x, y)=x y$.

(D) $\phi\left(d_{1}, \cdots, d_{n}\right)$

$$
\begin{aligned}
& =\min \left[\phi\left(d_{1}-r, \cdots, d_{a}-r\right)+\phi\left(d_{a+1}-s, \cdots, d_{b}-s\right)+\cdots\right. \\
& \left.\quad+\phi\left(d_{c+1}-t, \cdots, d_{n}-t\right)+\phi(r, s, \cdots, t)\right]
\end{aligned}
$$

where $r, s, \cdots, t$ range over all nonnegative integers such that the arguments are nonnegative.

Many other properties of $\phi$ can be derived from these four, including the following:

(E) $\phi\left(d_{1}, \cdots, d_{n}\right)=\min \left[\phi\left(d_{1}-t, \cdots, d_{n-1}-t\right)+t d_{n}\right]$.

(F) $\phi\left(d_{1}, \cdots, d_{n}\right)=\min \left[\left(d_{n-1}-T_{n-2}\right)\left(d_{n}-T_{n-2}\right)+\sum_{i=1}^{n-2} t_{i}\left(d_{i}-T_{i-1}\right)\right]$, where $T_{i}=t_{1}+\cdots+t_{i}$.

Property $(F)$ shows that $\phi$ is a solution of a quadratic integer programming problem. Some references are given at the close of this paper; others may be found in these listed publications.

We assume using (A) that the $d_{i}$ are numbered so that $d_{1} \leqq d_{2} \leqq$ $\cdots \leqq d_{n}$ and then use (C) and (E) to find an explicit expression for $\phi$.

Let $D=\left(d_{1}, \cdots, d_{n}\right)$. For $2 \leqq i \leqq n$ let $q_{i}=q_{i}(D)=\left(d_{1}+\cdots+d_{i}\right)$ $\cdot(i-1)^{-1}$ and let $k=k(D)$ be the smallest $i$ for which $q_{i}$ assumes its minimum value. Let integers $q=q(D)$ and $r=r(D)$ be defined by $d_{1}+\cdots+d_{k}=q(k-1)+r$ and $0 \leqq r<k-1$. Let $c_{i}=q-d_{i}$ for $i=1$, $\cdots, k$ and let $C=C(D)=\left(c_{1}, \cdots, c_{k} ; r\right)$. Let $s_{1}=c_{1}+\cdots+c_{k}$ and $s_{2}=\sum_{i<j} c_{i} c_{j}$. We define a function $f(D)$ by

Received by the editors April 3, 1965.

1 Work on this paper supported by a grant from the American Association of University Women. 


$$
f(D)=s_{2}+r s_{1}+[(r+1) r / 2]
$$

and show by induction on $n$ that $f(D)=\phi(D)$, i.e., $f(D)$ satisfies (E) and $(\mathrm{C})$.

If $d_{1}, \cdots, d_{n}$ are allowed to take on all real values, it can be shown easily by induction on $n$ that the function $\phi(D)$ satisfying $(C)$ and (E), with $t$ ranging over the real numbers, is

$$
\phi(D)=\sum_{i<j} d_{i} d_{j}-\frac{n-2}{2(n-1)}\left(d_{1}+\cdots+d_{n}\right)^{2} .
$$

Going back to the integer case, we note that $s_{1}=\left(q-d_{1}\right)+\cdots$ $+\left(q-d_{k}\right)=k q-[(k-1) q+r]=q-r$ and hence that

$$
q(D)=s_{1}(D)+r(D) \text {. }
$$

We also observe that $q_{i}$ is the average of the $i-1$ integers $d_{1}+d_{2}$, $d_{3}, \cdots, d_{i}$. Therefore one and only one of the following holds for each $i \geqq 3$ :
(a) $q_{i-1}<q_{i}<d_{i}$
(b) $q_{i-1}=q_{i}=d_{i}$,
(c) $q_{i-1}>q_{i}>d_{i}$.

Since the $d^{\prime}$ 's are nondecreasing, if $q_{j} \leqq q_{j+1}$ for some $j$ then (2) implies that $q_{j} \leqq q_{j+1} \leqq q_{j+2} \leqq \cdots \leqq q_{n}$. The first such $j$ must therefore be $k$. Hence we have

$$
q_{2}>q_{3}>\cdots>q_{k} \text { and } q_{k} \leqq q_{k+1} \leqq \cdots \leqq q_{n} .
$$

It follows from (2) and (3) that

$$
d_{i}<q_{i} \leqq q_{i+1} \leqq d_{i+1} \quad \text { if and only if } i=k .
$$

Since $(k-1) q+r=d_{1}+\cdots+d_{k}=(k-1) q_{k}$ and $0 \leqq r<k-1$, we also note that $q$ is the greatest integer $\left[q_{k}\right]$ in $q_{k}$.

In the case $n=2$, we always have $k=2, q=d_{1}+d_{2}, r=0$, $C=\left(d_{2}, d_{1} ; 0\right)$ and $f\left(d_{1}, d_{2}\right)=s_{2}=d_{1} d_{2}$. Hence $f\left(d_{1}, d_{2}\right)=\phi\left(d_{1}, d_{2}\right)$. We assume that $f(D)=\phi(D)$ for $n=m$ and show that it follows for $n=m+1$.

When $n=m+1$, we let $d_{m+1}=d, D^{*}=\left(d_{1}, \cdots, d_{m}, d\right)$, and $D=\left(d_{1}, \cdots, d_{m}\right)$. Using the hypothesis of the induction and (E) we see that $\phi\left(D^{*}\right)$ is

$$
\min [f(D-t I)+t d]
$$

where $t$ ranges over $1,2, \cdots, d_{1}$ and $D-t I$ denotes $\left(d_{1}-t, \cdots\right.$, $\left.d_{m}-t\right)$.

Let $F(t)=f(D-t I)+t d$ and let $\nabla F(t)=F(t)-F(t-1)$. Clearly $\nabla F(t)=d-\Delta f(D-t I)$ where $\Delta f(D)=f(D+I)-f(D)$. We first prove the following: 
Lemma. $q(D+I)-q(D)$ is 1 if $r(D+I)>0$ and is 2 if $r(D+I)=0$, $\Delta f(D)=q(D)+1$, and $\nabla F(t)=d-q(D-t I)-1$.

Proof. Let $C(D)=\left(c_{1}, \cdots, c_{k} ; r\right)$. When $k(D+I)=k(D)$, it is clear that if $r<k-2$ then $q(D+I)=q(D)+1$ and that $C(D+I)$ $=\left(c_{1}, \cdots, c_{k} ; r+1\right)$ while $q(D+I)=q(D)+2$ and $C(D+I)=\left(c_{1}+1\right.$, $\left.\cdots, c_{k}+1 ; 0\right)$ if $r=k-2$. In each of these cases, one calculates that $f(D+I)=f(D)+s_{1}(D)+r(D)+1$. This and (1) lead to $\Delta f(D)=q(D)$ +1 . Then $\nabla F(t)=d-\Delta f(D-t I)=d-q(D-t I)-1$.

Next we note that

$$
q_{i}(D+I)=q_{i}(D)+1+1 /(i-1) .
$$

Since $1 /(i-1)$ is a decreasing function of $i$ for $i \geqq 2$, this implies that $k(D+I) \geqq k(D)$. Hence the only case remaining is that in which $k(D+I)>k=k(D)$. We then have

$$
q_{k+1}(D) \geqq q_{k}(D), \quad q_{k+1}(D+I)<q_{k}(D+I) .
$$

Using (6) and (7) we obtain

$$
\begin{aligned}
0 & <q_{k}(D+I)-q_{k+1}(D+I) \\
& =\frac{1}{k-1}-\frac{1}{k}-q_{k+1}(D)-q_{k}(D) \leqq \frac{1}{(k-1) k} .
\end{aligned}
$$

Since $q_{k+1}$ and $q_{k}$ are both expressible as integers divided by $(k-1) k$, (8) implies that $q_{k}(D+I)-q_{k+1}(D+I)=1 /(k-1) k$ and

$$
q_{k+1}(D)=q_{k}(D) \text {. }
$$

One sees from (9) and (2) that $d_{k+1}=q_{k}(D)$. One similarly finds that $d_{i}=q_{k}(D)$ for $k<i \leqq k(D+I)$.

Since $q_{k}(D)=d_{k+1}$ is an integer, we have $q(D)=q_{k}(D)$ and $r(D)=0$. Hence $d_{i}=q(D)$ for $k+1 \leqq i \leqq k(D+I)$ and it is easily seen that $q(D+I)=q(D)+1, r(D+I)=r(D)+1=1$, and $C(D+I)=\left(c_{1}, \cdots\right.$, $\left.c_{k}, 0, \cdots, 0 ; 1\right)$. One now calculates that $f(D+I)-f(D)=s_{1}(D)+1$ $=q(D)+1$. As before, $\nabla F(t)=d-q(D-t I)-1$, which completes the proof of the lemma.

It follows from the lemma that $\nabla F(t)$ increases by 1 or 2 and $q(D-t I)$ decreases by 1 or 2 when $t$ increases by 1 .

We prove that $f\left(D^{*}\right)=\phi\left(D^{*}\right)$ by considering cases. First let $d \geqq q(D)$. For $1 \leqq t \leqq d_{1}$, we then have $d \geqq q(D)>q(D-t I)$ and so $\nabla F(t)=d-q(D-t I)-1 \geqq 0$. Hence the minimum $\phi\left(D^{*}\right)$ of the $F(t)$ is $F(0)=f(D)$. If $k\left(D^{*}\right)>k(D)$, we must have $k(D)=m$ and $d \geqq q(D)$ implies that $C(D)$ and $C\left(D^{*}\right)$ are of the form $\left(c_{1}, \cdots, c_{m} ; r\right)$ and 
$\left(c_{1}, \cdots, c_{m}, 0 ; r\right)$ respectively. If $k\left(D^{*}\right)=k(D), C\left(D^{*}\right)=C(D)$. Either way, $f\left(D^{*}\right)=f(D)=\phi\left(D^{*}\right)$.

It remains to consider $d<q(D)$. Then $d_{m} \leqq d<q(D) \leqq q_{k}(D)$ and (4) implies that $k(D)=m$ and $k\left(D^{*}\right)=m+1$. Now $d_{1}>0$ since $d_{1}=0$ would imply $k\left(D^{*}\right)=2$, which is less than $m+1$ for $m \geqq 2$. Then $d \geqq d_{2}>d_{2}$ $-d_{1}=q\left(D-d_{1} I\right)$. Hence $d-q(D-t I)$ must change sign as $t$ goes from 0 to $d_{1}$ in one of the two following ways:

First let there be a $c$ with $0<c<d_{1}$ such that $d=q(D-c I)$. Then $\nabla F(t) \leqq-1$ for $t \leqq c$ and $\nabla F(t) \geqq 0$ for $t>c$. Hence $\phi\left(D^{*}\right)=F(c)$ $=f(D-c I)+c d$. We let $k=k(D-c I)$ and show that $k=m$. Since $c>0$,

$$
q_{k}(D-c I) \leqq d_{k+1}-c<d=q(D-c I) \leqq q_{k}(D-c I)
$$

would be a contradiction unless $k=m$ (and $d_{k+1}$ does not exist). Now it is clear that $q\left(D^{*}\right)=q(D-c I)+c$. Letting $C(D-c I)=\left(c_{1}, \cdots\right.$, $\left.c_{m} ; r\right)$, one has $C\left(D^{*}\right)=\left(c_{1}, \cdots, c_{m}, c ; r\right)$ and $f\left(D^{*}\right)=f(D-c I)$ $+c\left(c_{1}+\cdots+c_{m}\right)+c r=f(D-c I)+c d=\phi\left(D^{*}\right)$.

Finally let there be a $c$ with $0 \leqq c<d_{1}, d=q[D-(c+1) I]+1$, and $d=q(D-c I)-1$. Then $\nabla F(t) \leqq 0$ for $t<c$ and $\nabla F(t) \geqq 2$ for $t \geqq c$. Hence $\phi\left(D^{*}\right)=F(c)=f(D-c I)+c d$. Since $q(D-c I)-q[D-(c+1) I]$ $=2$, the lemma tells us that $r(D-c I)=0$. As before, $k(D-c I)=m$. Let $C(D-c I)=\left(c_{1}, \cdots, c_{m} ; 0\right)$. One then calculates that $q\left(D^{*}\right)$ $=q(D-c I)+c-1, \quad C\left(D^{*}\right)=\left(c_{1}-1, \cdots, \quad c_{m}-1, \quad c ; m-1\right), \quad$ and $f\left(D^{*}\right)=f(D-c I)+\left(c_{1}+\cdots+c_{m}-1\right) c=f(D-c I)+d c=\phi\left(D^{*}\right)$. This completes the proof.

\section{BIBLIOGRAPHY}

1. E. M. L. Beale, On quadratic programming, Naval Res. Logist. Quart. 6 (1959), 227-243. 1957.

2. R. Bellman, Dynamic programming, Princeton Univ. Press, Princeton, N. J.,

3. - Mathematical optimization techniques, Univ. of California Press, Berkeley, Calif., 1965.

4. R. E. Gomory, An alogrithm for integer solutions to linear programs, Recent advances in mathematical programming, pp. 269-302, McGraw-Hill, New York, 1963.

5. - Mathematical programming, Slaught Memorial Paper No. 10, pp. 99 110, Math. Assoc. Amer., Buffalo, N. Y., 1965.

6. A. P. Hillman, D. G. Mead, K. B. O'Keefe and E. S. O'Keefe, Ideals generated by products, Proc. Amer. Math. Soc. 17 (1966), 715-718.

7. H. P. Kunzi and W. Oettle, Integer quadratic programming, Recent advances in mathematical programming, pp. 303-308, McGraw-Hill, New York.

8. P. Wolfe, The simplex method for quadratic programming, Econometrica, 27 (1959), 382-398.

University of Santa Clara and

University of Washington, Seattle 